\title{
Facilitating Culturally Diverse Groups with Visual Templates in
}

\section{Collaborative Systems: Increasing Structuration to Improve Precision}

\begin{abstract}
Purpose - The use of visual templates has proven instrumental in supporting group meetings. This study explores whether visual templates enable culturally diverse groups to achieve greater task precision in face-to-face meetings.

Design/methodology/approach - Building on Adaptive Structuration Theory (AST), it is argued that visual templates provide structuration for face-to-face meetings, even more so when they are embedded in computer-supported collaborative systems. In particular, it is hypothesized that the higher the degree of structuration imposed by visual templates, the higher the degree of task precision will be. It is also hypothesized that this relationship is positively moderated by group cultural diversity: higher cultural diversity will further sustain the positive effects of visual templates that provide higher structuration.
\end{abstract}

Findings - Results of an experiment with 229 managers from 49 countries confirm that facilitating groups with visual templates embedded in a computer-supported collaborative system significantly increases task precision at high levels of cultural diversity.

Research limitations/implications - This study contributes to Positive Organizational Scholarship (POS) by investigating the use of visual templates as a contingency factor that increases performance - specifically task precision - of co-located, culturally diverse groups.

Practical implications - Results indicate that visual templates embedded in a computersupported collaborative system are an effective method for increasing task precision in face-to-face meetings of culturally diverse groups. 
Originality/value - Theories from information systems and visualization are integrated into cross-cultural management with a view to sustaining the effectiveness of culturally diverse groups. The study sample is characterized by highly culturally diverse groups interacting in face-to-face meetings.

Keywords: multicultural groups, cultural diversity, visual templates, face-to-face meetings, computer-supported collaborative systems, structuration theories. 


\section{Introduction}

In an increasingly multicultural work environment, finding best practices for facilitating face-toface meetings emerges as a particularly urgent research gap. Multicultural groups face greater challenges as compared to homogeneous groups (i.e., groups composed of members from the same cultural background): differences in communication practices, working styles and language threaten collaboration effectiveness (Meyer, 2014). However, if adequately supported, culturally diverse groups (which we use as synonymous with "multicultural groups") may outperform culturally homogeneous groups, thanks to their varied backgrounds (Adler, 2003; McLeod et al., 1996). In consistence with the perspective of Positive Organizational Scholarship, this study aims to advance the means which can support organizational group work and, in particular, the performance of culturally diverse groups. It contributes by proposing novel means, namely the use of visual templates to structure group discussions in face-to-face meetings.

Visuals are increasingly used in organizations, as testified by the widespread diffusion of diagrams, visual metaphors (Eppler and Platts, 2009) and timelines (Yakura, 2002). Visual facilitation, in particular, is the use of visual templates to support face-to-face meetings, in which one person acts as a facilitator and moderates the discussion by documenting the participants' inputs to the templates themselves (Eppler and Burkhard, 2007). Visual templates (e.g., BCG matrix, SWOT diagram, Porter's five forces diagram) provide a graphic structure onto which knowledge, in the form of text and images, can be meaningfully mapped. As argued by Meyer and colleagues: "With the unprecedented rise in the use of visuals, and its undeniable omnipresence in organizational context, as well as in individuals' everyday life, organization and management science has recently started to pay closer attention to the to date under-theorized "visual mode" of discourse and meaning construction" (Meyer et al., 2013, p. 489).

Current literature portrays visual facilitation as a "panacea" for increasing performance in group work (Buzan, 2004; Roam, 2009), but has paid less attention to the cultural characteristics of the groups using visual templates. In general, the literature assumes that visual templates will have the 
same effect regardless of the discussants' backgrounds. However, this assumption does not seem accurate since group dynamics change in the presence of cultural diversity (Mach and Baruch, 2015). As Berry (2015, p. 343, based on Campbell, 1959) argues: "all theories are valid, under some conditions. Hence, the conditions (often social and cultural in nature) need to be examined in a wide variety of contexts in order to discern the validity of theories that we have developed in our own cultures." There is a need to understand whether visual templates can be of help for culturally diverse teams, and, if so, under what conditions.

Another limitation of current literature consists of focusing on the message, while neglecting the medium used for visual facilitation. To date, most scholars have investigated the effects of visual templates with analogic media - such as, for example, with paper and pencil. Yet organizations are increasingly using new technologies to facilitate meetings; and visual templates are therefore utilized with both non-digital and digital media. This difference is not trivial, as diverse media impose different degrees of structuration to group interaction. According to Adaptive Structuration Theory, structuration emerges in human action, as people interact with technologies (DeSanctis \& Poole, 1994). In particular, digital media such as computer-supported collaborative systems ${ }^{1}$ provide higher structuration than non-digital media (Silver, 2008), insofar as they leave less room for interpretation in the way they can be used.

In this paper, it is argued that visual templates can be particularly helpful in dealing with the communication problems of culturally diverse groups for the reason that they provide a support to structure and document the conversation. It is hypothesized that visual templates providing higher structuration will lead to increased task precision (as compared to visual templates providing lower structuration). This is the case, since higher structuration is more likely to impose a faithful use (Poole and DeSanctis, 1990) of visual templates, and hence to enable users to actually experience the benefits of visual facilitation. It is also hypothesized that cultural diversity will positively moderate the relationship between structuration and task precision: Higher cultural diversity will

\footnotetext{
${ }^{1}$ Computer-supported collaborative systems are software environments specifically developed to be utilized in a group.
} 
further sustain the positive effects of visual templates that provide higher structuration. In the presence of high structuration, culturally diverse groups will be able to productively leverage their differences in cognitive schemas (Markus and Kitayama, 1991; Leung and Morris, 2015) and norms (Norenzayan et al., 2002; Leung and Morris, 2015).

Task precision is an aspect of group performance. It is the extent to which groups are effective and efficient in providing solutions to the task at hand. Highly precise groups provide solutions that are viable (effectiveness) without making wrong or irrelevant attempts (efficiency). Task precision is important in contemporary organizations, which are competing not just in innovation, but also in their swiftness to respond to changing and disruptive environments. It is also more specific than the broader construct of "group performance" and hence it is more convenient to operationalize (see also Mell et al., 2014). By focusing on a specific aspect of group performance, this paper aims to provide more precise information regarding the extent to which culturally diverse groups can be sustained. In so doing, it also addresses the concerns of Mathieu et al. (2008), who noted that the tendency to focus on overly broad constructs (such as group performance) has led to different operationalizations, and in turn to inconsistent results in the literature on groups and teams.

This paper focuses on the following research question: Does the structuration imposed by a visual template lead to increased task precision? Is this relationship moderated by the degree of group cultural diversity? To address this, an experimental study was conducted with over two hundred managers, from all continents, enrolled in MBA programs. Each group, which consisted of four members on average, was exposed either (1) to a condition providing higher structuration (a visual template embedded in a computer-supported collaborative system), or (2) to a condition providing lower structuration (the same visual template printed on paper), or (3) to a control condition with no structuration (plain paper with no visual template). Visual templates embedded in computer-supported collaborative systems provide greater structuration than paper-based visual templates, since the digital medium offers less flexibility for users to appropriate the structures given by the system. The moderating variable of cultural diversity is defined - for the purposes of 
this study - as the multiplicity of country of origin within each group, clustered into global macro regions in order to account for cultural macro variations (Collard and Foley, 2002; Nisbett, 2003; Leung and Morris, 2015).

To preview the results, confirmation is found of a significant main effect of visual templates on task precision, and of a significant moderating effect of group cultural diversity. This work contributes to research on cross-cultural management by articulating how cultural diversity positively moderates the effects of using visual templates in the context of face-to-face group meetings. In particular, it advances the perspective of Positive Organizational Scholarship (POS) (Stahl and Tung, 2015): While most scholars have focused on the negative effects, POS emphasizes the "bright side" of cultural diversity (Stahl et al., 2010a) and it sets out to understand the enablers of effective group processes and outcomes, so as to allow organizations to capitalize on the richness of cultural diversity. This contribution is developed by taking an interdisciplinary perspective: insights from Adaptive Structuration Theory and related fields of information systems and visualization are leveraged to find novel means of sustaining culturally diverse groups.

An additional value of this work consists of studying groups characterized by high cultural diversity, using a pan-continental sample of 229 subjects, from 49 countries, with managerial work experience: highly multicultural and co-located groups are rarely studied in management research. In the presence of such a diverse sample, cultural diversity is aptly operationalized according to the concept of Geography of Thought (Nisbett, 2003), which establishes that cultural differences are experienced across macro-regions, rather than across nations. This ensures the possibility of making aggregations that capture cultural diversity at the team level, while also preventing the risk of inflating the measure by assessing micro differences at national level.

Implications for managerial practice are straightforward: when in the presence of culturally diverse groups, visual templates should be utilized and embedded in a collaborative system to provide structure and guidance for the discussion. The results indicate that the most commonly used 
means for facilitating meetings in organizations, such as plain flipcharts or sticky notes, may be suboptimal in the presence of a highly heterogeneous range of cultural backgrounds.

This article is organized as follows: the next section provides an overview of extant literature and builds the hypotheses. In the following section, the experimental methodology is explained in detail. The fourth section provides the statistical results, and is followed by a section that discusses such results. The paper concludes with implications for theory and practice.

\section{Literature Review}

\section{Visual templates and collaborative systems}

Visual templates are increasingly utilized in organizations to support communicative and collaborative tasks, to the point that they are being recognized as "genres" (Yates and Orlikowski, 2007). According to Meyer et al. (2013, p. 495), the visual mode "is characterized by a prevalence of holistic and immediate information, rather than linear and sequentially arranged information". The benefits of visual templates, as compared to purely textual or verbal communication modes, are to be found also in their structure, which offers a "representational guidance" (Suthers, 2001). According to Suthers (2001, p. 2), representational features of a template utilized by a group have significant effects on the discussants' discourse, since "representational guidance constrains which knowledge can be expressed in the shared context, and makes some of that knowledge more salient and hence a likely topic of discussion".

A visual template provides a graphical structure onto which information and knowledge can be meaningfully mapped, related, and put into perspective (Eppler and Platts, 2009). This graphical structure, made up of arrows, boxes and labels, offers "affordances" (Demir, 2015; Gibson, 1979; Jarzabkowski and Kaplan, 2015) - i.e., cues as to how the visual template should be used in practice. For example, empty boxes on a strategy map afford the filling in of strategic issues, whereas arrows afford reflection on relationships between such issues. Timelines (Yakura, 2002) help planning and coordinating efforts, while mind maps (Buzan, 2004) are widely utilized for 
brainstorming and note-taking during meetings. They enable placing the discussion topic in a balloon at the center of the paper, and branching out topics and sub-topics as they emerge during the conversation. When ideas are mapped, it is easier for group participants to identify differences between their mental models and the concepts being visualized (Schnotz and Kurschner, 2008). The use of visual templates can increase the quantity and quality of ideas developed in group work; as well as the number of ideas being recalled by group members (Perez Garcia and Bresciani, 2015).

Visual templates have proven useful for group work not only in analogic format (such as on paper and posters) but also in digital formats, through the use of computer-supported collaborative systems (Suthers, 2001; Hundhausen, 2005; Bresciani and Eppler, 2009), generally referred to as “collaborative systems". These are software applications designed to support co-located and remote collaboration in organizations. As ICT is nowadays widespread in organizations, visual templates are progressively utilized in digital formats for supporting collaborative tasks (Isenberg et al., 2011) and decision making (Wheeler and Valacich, 1996; Al-Kassab et al., 2014; Geraldi and Arlt, 2015).

A collaborative system offers further guidance for group discussion, by providing structures that assist the use of the visual templates - such as, for example, the drag and drop feature that enables placing branches in a mind map. As noted by Watson et al. $(1994$, p. 48), a collaborative system "gives a group the opportunity to change its existing behavior because it presents the group with new structures that can be adopted". The structures provided by collaborative systems have been extensively studied and discussed in the context of Adaptive Structuration Theory (AST) (Poole and DeSanctis, 1990). AST is one of the most widely utilized theories in the field of information systems - "perhaps the most influential" of all theories deployed in the field, according to Jones and Karsten (2008, p. 127). It is robust to empirical testing (Etudo et al., 2015) and has been used in recent studies of both face-to-face and virtual groups (Niederman et al., 2008; Naik and Kim, 2010).

AST accounts simultaneously for the effects of the collaborative system and of the individuals who interact with such a system. Adaptive structuration occurs when the collaborative system is 
brought into use and is then appropriated in social action (DeSanctis and Poole, 1994). The effectiveness of collaborative systems vis-à-vis their intended purpose is related to the more or less faithful appropriation of the system. When users appropriate the collaborative system in the manner expected and envisioned by its developers, the appropriation of the system is deemed faithful. Conversely, users can re-appropriate the collaborative system by using it in a manner which is unfaithful, that is, different from the intended usage. When a system provides low structuration, there are greater chances that users will re-appropriate it in unintended - and thus less effective ways. Conversely, when a system provides high structuration, there are fewer opportunities for users to overcome the constraints posed by the system itself (Wheeler and Valacich, 1996; Alexander et al., 2015). As a consequence, there are also fewer meta-discussions and conflicts regarding how to structure the conversation.

This study proposes that groups using visual templates embedded in a collaborative system will exhibit greater precision in performing the task at hand, meaning that they will come to develop a viable solution with fewer failed attempts. This should be the case since the visual templates provide a graphical structure around which group members can develop their task solutions, thus minimizing the search for relevant categories and relationships. Furthermore, the use of a collaborative system will enforce the appropriation of the visual template in the 'correct' manner, therefore enabling groups to benefit the most from its ensuing structuration. The group will likely use the structures offered by the visual templates and engage in discussion about the development of solutions for the task at hand (rather than in meta-discussions about how to approach the task itself). Therefore it is hypothesized that:

H1: The higher the degree of structuration imposed by visual templates, the higher task precision will be.

The moderating effect of group cultural diversity 
Cultural diversity may have both positive and negative influences on group performance (Milliken and Martins, 1996; Williams and O'Reilly, 1998; van Knippenberg et al., 2004; Adler, 2003; Zoogah et al., 2011): it can increase productivity (Stahl et al., 2010a), creativity (McLeod et al., 1996), and the quality of decision making (Shachaf, 2008), but may also hinder communication (Adler, 2003), trigger conflict, and decrease social integration (Stahl et al., 2010b). Advantages of cultural diversity can be substantial: in a field study, $\mathrm{Ng}$ and Tung (1998) found that culturally diverse organizations have higher organizational effectiveness, in particular with increased profitability. In a more recent study, Nielsen and Nielsen (2013) consistently found that a top management team's cultural diversity is positively related to firm performance.

Yet culturally diverse groups face greater difficulties compared to culturally homogeneous groups due to miscommunication, mistrust and lack of cohesion (Mach and Baruch, 2015). These 'problems of understanding' are often caused by differences in values (Hofstede, 2001; House et al., 2013), cognitive structures (or "schemas", Markus and Kitayama, 1991; Leung and Morris, 2015) and norms (Norenzayan et al., 2002; Leung and Morris, 2015), which are found across cultures. For instance, Westerners rely mostly on rules, categorizations and formal logic, whereas East Asians rely mostly on intuitive associations and similarities (Nisbett, 2003): this difference does not signal different capabilities but rather different norms regarding what is considered to be more important in analyzing a given situation (Leung and Morris, 2015).

Further evidence indicates that - in an increasingly globalized world - cultural diversity remains particularly strong across macro regions of the world, and especially along the axes of North and South, and East and West (Levine, 2008; Nisbett, 2003; Pattanaik, 2016). Nisbett (2003) suggests that cultural diversity is produced by differences in historical, religious and philosophical traditions, which laid the foundations for the emergence of a 'Geography of Thought'. For example, the early work of Greek philosophers is considered to be at the roots of a 'European Thought', which favors linear thinking and a rational view on the nature of humanity (Snell, 1953). Additional research by Collard and Foley (2002) suggest that human culture exhibit 'strong geographical patterns', and 
accordingly identified environmental determinants of cultural diversity. It finds that similarities in temperature and rainfall produce cultural commonalities across neighboring countries (Collard and Foley, 2002).

In the field of collaborative systems, group cultural diversity has been studied predominantly in the context of virtual groups (Daily et al., 1996; Paul et al., 2004; Zhang et al., 2007; Shachaf, 2008). For instance, Gray and Olfman (1989) tested the effectiveness of a collaborative system that produced automatic translations among the virtual group participants' languages. Shigenobu et al. (2007) explored the efficacy of an annotation function for virtual intercultural communication. Daily et al. (1996, p. 281) discovered that culturally diverse groups using Group Decision Support System (GDSS) "produced a significantly higher number of non-redundant, realistic ideas than homogeneous groups that used the GDSS".

Research on cultural diversity (in both management and information systems) focuses almost exclusively on textual or verbal communication, even though individuals communicate through different modes (e.g., verbal, visual, and gestural). It has been theorized (but not yet empirically tested) that culturally diverse groups may experience a number of benefits from the use of visuals (Bresciani, 2013). Yet individuals from diverse cultures might have different opinions regarding how to utilize visual templates, since they have different cognitive structures (Markus and Kitayama, 1991; Leung and Morris, 2015) and norms (Leung and Morris, 2015). In a similar way, collaborative systems may be perceived as affording different possibilities, depending on the values and beliefs of individual users (Vyas et al., 2016). Hence, the effect of visual templates on task precision may vary at different levels of cultural diversity. In particular, visual templates providing different degrees of structuration may produce different levels of task precision, depending on the cultural diversity of the groups in which they are used.

This paper advances that cultural diversity positively moderates the relationship between the degree of structuration of visual templates and task precision. It is expected that high cultural diversity will further increase the positive effects of visual templates offering higher structuration. 
In other words, when a group uses visual templates that impose a high degree of structuration, high cultural diversity will strengthen the positive relationship between use of visual templates and task precision. The moderating mechanism of cultural diversity is expected to work as follows. In the presence of low structuration, high cultural diversity will exacerbate differences in cognitive styles and values. These differences will result in off-topic discussions about how to appropriate the structures provided by the visual templates; therefore reducing the positive effects of visual templates on task precision. In the presence of high structuration, culturally diverse groups will be able to productively leverage their differences in cognitive styles and values, so as to deliver solutions that are both effective and efficient in addressing the task at hand (task precision). In this case, the positive effects of high cultural diversity (as theorized by POS) will compound with the positive effects of high structuration (as theorized by AST). The following hypothesis is advanced:

H2. Group cultural diversity will positively moderate the relationship between the degree of structuration imposed by visual templates and task precision. Higher cultural diversity will further sustain the positive effects of visual templates that provide higher structuration.

The resulting research model is displayed in Figure 1.

Insert Figure 1 about here

\section{Methodology}

The research model is tested through a lab experiment, which is considered a powerful yet underutilized methodology in cross-cultural management (van Witteloostuijn, 2015). The experiment is based on a between-subject design: each subject is exposed to only one condition. It comprises two experimental conditions: (1) a lower-structuration condition in which groups received task-specific visual templates printed on A3 papers and (2) a higher-structuration condition in which groups received the same templates embedded in a collaborative system. These 
experimental conditions are compared to a control condition in which groups received the same task but no visual templates (this is a standard procedure in experimental research to test the effect of the experimental manipulation; see Shadish et al., 2002). In the next paragraphs, we describe the visual templates used in the experimental conditions, as well as the experimental task and procedure.

\section{Visual templates and experimental conditions}

Subjects in the experimental conditions are asked to document their discussion on visual templates designed for the task of exploring strategic alliances (see next paragraph for a discussion of this task). The "competence complementarity chain" (adapted from Pietroforte, 1996) aims to support the identification of complementarity areas (for an example, see Figure 2). The "innovation opportunity map" (adapted from Muller and Välikangas, 2002) assists the exploration of joint innovation via the systemic recombination of the partners' competences (for an example, see Figure 3). In both experimental conditions, subjects receive the same visual templates, the only difference being the degree of structuration. Groups in the lower-structuration condition receive the visual templates printed on an A3 sheet of paper together with sticky notes and pens, while groups in the higher-structuration condition use the same visual templates within a collaborative system (called Let's Focus). Each group in the higher-structuration condition has one computer which is connected to a screen and a wall projector, enabling all participants to see annotations made on the visual template.

Control groups are not provided any visual templates, but they receive the same instructions as the experimental groups - which include the labels and categories of the visual templates (such as 'competences', 'market needs', 'innovation opportunities'). Such labels are provided in the form of agenda items for the group to structure their meeting (e.g., 'make your company's competences explicit', 'explore joint innovation opportunities'). In this way, control groups are in a fair position compared to experimental groups, and the observed differences can be attributed to the intervention variable (i.e., the visual templates). 


\section{Experimental task and procedure}

The participants are randomly grouped and assigned to one of the three conditions. A hidden-profile case study is used to simulate an organizational meeting (Stasser and Titus, 2003; Schulz-Hardt et al., 2006) on the topic of strategic alliances (Comi et al., 2009). This context is appropriate, since cultural differences affect not only the type of alliance choice, but also managerial aspects such as the negotiations involved in the alliance (Shenkar et al., 2008; Stahl and Tung, 2015). To simulate an alliance meeting, half of the participants in each group are given detailed information about their own organization, but only limited information about the alliance partner. This results in half of the participants reading one version of the case, and the other half of the participants reading the second version of the case.

As experimental task, participants are asked to identify complementary competences between the two organizations and to foresee opportunities for collaborative innovation. In order to reach this goal, they have to share their respective (asymmetric) knowledge. To avoid contamination between groups and across conditions, each group is convened to a meeting room or private space in which they can discuss the strategic alliance between their companies. All groups are allowed one hour to complete the task, and are asked to document the discussion with the material received from the experimenters, which differs depending on the condition (see above paragraph.). At the end of the experiment, group members fill out a questionnaire which includes demographic information and additional questions for control purposes (see paragraph below). Afterwards, a plenary debriefing with discussion is held with participants from each condition. The post-experiment survey is carried out before the debriefing so as to avoid any contamination in the participants' answers.

\section{Measures}

The case study used in the experiment has a sole objective solution, which is reported and detailed in the accompanying teaching note (Comi et al., 2009). This makes the evaluation of the groups' 
outcome straightforward. To measure task precision, we count the number of items identified by the groups. Secondly, we divide the number of correct items by the number of total items documented by the groups. Items are defined as both keywords and short strings of text documented in the visual templates or flipcharts: if they correspond to items in the case solution, they are considered "correct" items. This is in line with the procedure used by Mell et al. (2014 pg. 1162): "Team performance scores reflected the quality of the team's decisions and [is] based on the similarity of their solutions to the objectively correct solution." A cautionary approach is adopted so that the item is not counted if it is not a clear match with the items reported in the case study solution. A high degree of task precision suggests that the group solution has been achieved without the conversation digressing into irrelevant details. Figure 2 shows an example of a competence complementarity chain template filled in by a group in the paper-based condition. Figure 3 shows an example of an innovation opportunity map populated by a group in the collaborative system condition.

Insert Figure 2 and 3 about here

In a post-experiment questionnaire, participants are asked about their country of origin. Group cultural diversity is calculated with an index based on seven categories corresponding to macro regions of the World (Europe, Asia, Middle East, North America, Latin America, Africa, Oceania). The calculation involves aggregating individual countries within world regions, and producing Gibbs-Martin indices of diversity at the group level (Gibbs-Martin indices had already been utilized by Blau (1977) to calculate cultural diversity). Following Gibbs and Martin (1962), the index of diversity is computed as follows: $\mathrm{D}=1-\sum_{\mathrm{i}=1}^{\mathrm{N}} \mathrm{p}_{\mathrm{i}}^{2}$, where $\mathrm{N}$ is the number of categories and $\mathrm{p}$ is the proportion of individuals within each category. In order to compare diversity across groups of different sizes, normalized indices are computed as follows: $\mathrm{D}_{\mathrm{n}}=\mathrm{D} * \mathrm{~K} /(\mathrm{K}-1)$, where $\mathrm{K}$ is the number of individuals within the group. The calculated cultural diversity of a group is a continuous 
variable ranging from 0 (when all group members are from the same world region) to 1 (when all group members are from different regions).

We explain our choices of using country of origin, and aggregating to macro regions as follows. Firstly, country of origin is commonly used to calculate cultural diversity (Mach and Baruch, 2015). Taras and colleagues reviewed "half a century" of approaches in measuring culture and found that $79 \%$ of the studies they analyzed "used country as a proxy for culture" (Taras et al., 2009, p. 358). While more sophisticated instruments for measuring cultural differences are available (e.g., Hofstede's or GLOBE's comparative cultural frameworks) their application would not have been feasible in the context of this experimental study. Hofstede's (Hofstede, 2001) and GLOBE's (House et al., 2013) comparative cultural frameworks, in fact, require a sample of at least 20 subjects per culture (e.g., Hofstede et al., 2008, pg. 2), and hence are suitable to assess cultural differences across large groups. Our sample, on the contrary, comprises 229 subjects from 49 nations working within small groups.

Secondly, the choice of aggregating countries of origins was informed by an understanding that in an increasingly globalized world - cultural differences remain strong at the level of macro regions (e.g., Levine, 2008; Nisbett, 2003; Pattanaik, 2016), rather than of national countries (Tomlinson, 1999). In particular, calculating a diversity index based on countries of origin would have inflated the measure of group cultural diversity, because our sample is characterized by individuals coming from 49 different countries.

\section{Control variables}

A number of factors pertaining to the attitudes, roles, and demography of participants may influence the outcome variables of our study, therefore confounding the effects of visual templates. To rule out alternative explanations, we controlled for gender, age, experience of strategic alliances, level of English language, preference for group work (based on Campion et al., 1993), preference for visualization, facilitator's skillfulness and number of group participants. These control variables are 
measured through a post-experiment questionnaire conducted immediately after the experiment. Collecting such answers before the experiment could bias subjects' behavior while performing the experimental task, by prompting assumptions about the purpose of the study. This would have been problematic, since we conduct a between-subject experiment - i.e., an experimental design in which participants are exposed to only one condition, and are blind to the other conditions. We have also mitigated the issue of biased answers to control questions, by choosing or developing instruments whose items require respondents to think about their general experience (thus shifting attention away from participation in the experimental study).

\section{Sample characterization}

The experimental sample consists of 229 experienced managers enrolled in Executive Master in Business Administration (MBA) programs in Switzerland, of which 62\% are male. The median age is 32. Subjects are nationals from 49 different countries across all seven global regions (as defined in the previous section) - 162 subjects from Europe, 22 from Asia, 8 from the Middle East, 6 from North America, 16 from Latin America, 1 from Oceania and 8 from Africa. This provides an adequately culturally diverse sample to test the moderation of group cultural composition in our research model.

Participants work in groups of 4 on average, and exceptionally in groups of 3 subjects (2 groups), 5 subjects (1 group) or 6 subjects ( 2 groups) to accommodate the uneven number of participants. In total, 56 groups are formed (19 for the control condition, 20 for the paper-based condition and 17 for the collaborative system condition): The sample characteristics are fairly distributed across the three experimental conditions. In total, there are 21 culturally homogeneous groups (in which all group members are from the same region of the world), 2 groups in which two regions of the world are represented, 22 groups in which 3 regions of the world are represented and 11 groups in which each subject is from a different region of the world. 


\section{Results}

Analysis

The proposed research model can be tested with a laboratory experiment (Shadish et al., 2002) and analyzed with Analysis of Variance (ANOVA), a technique suitable for comparing more than two experimental conditions and particularly for testing moderation (Baron and Kenny, 1986; Field, 2009). The methodology and analysis follow state of the art techniques, such as the one employed by Mell et al. (2014) for moderation analysis.

Correlations among all variables (research model and control variables) are reported in Table 1, while Table 2 reports the means comparison for visual support and cultural diversity. An ANOVA is performed (Table 3, Figure 4): the statistical analysis shows the significant main effect of structure imposed by the support $(\mathrm{F}=8.488, \mathrm{p}=.016)$ and a non-significant effect of cultural diversity $(\mathrm{F}=0.741, \mathrm{p}=.660)$; however - as reported by Judd et al. (2014) - caution should be taken in interpreting the main effects when in the presence of a significant interaction. The analysis indicates a highly significant moderating effect of group cultural diversity on the impact of visual support on task precision $(\mathrm{F}=7.432, \mathrm{p}<.001$, two tailed) as reported in Table 3 . The resulting moderation is pictured in Figure 4: as it can be seen in the graph, the visual support leads to different performances when groups are culturally homogeneous or culturally diverse. To analyze these differences, a comparison of the conditions is carried out by analyzing planned contrasts (Table 4): this reveals a significant difference between the control condition and the paper-based condition, and also between the paper-based and the collaborative system conditions. A detailed discussion is provided in the following section.

Insert Table 1, 2, 3 and 4 about here

Insert Figure 4 about here 
To test if there is any effect of the control variables on the model, ANCOVA is performed by including all control variables simultaneously in the original model: age, gender, experience with alliances, level of English, preference for group work, preference for visualization, facilitator's skillfulness and number of group participants (Table 5). The results show that effect of the moderation is robust and remains highly significant $(\mathrm{F}=5.219, \mathrm{p}<.001)$ even when control variables are added to the model.

Insert Table 5 about here

\section{Findings}

This study offers two main findings: (1) utilizing visual templates for facilitating face-to-face group discussion leads to increased task precision; and (2) cultural diversity produces different effects on task precision, depending on the structuration offered by the visual template. Elaboration on these findings continues in the paragraphs below.

Visual templates lead to higher precision. As postulated in $\mathrm{H} 1$, visual templates, by providing higher structuration, were found to lead to increased task precision. Groups utilizing a visual template with collaborative systems (higher structuration) outperform groups utilizing paper-based visual templates (lower structuration). In general, groups using visual templates outperform groups using no visual templates to address the task at hand (as confirmed by the contrasts between the experimental conditions and the control condition).

Visual templates have different effects depending on the degree of group diversity. As predicted by $\mathrm{H} 2$, for culturally diverse groups the increase in task precision is sustained when visual templates are deployed within a collaborative system, but not when the same templates are paper-based and filled out manually. A visual support in paper format significantly improves task precision for homogeneous groups, but this advantage decreases as cultural diversity increases - leading to a 
paradoxical effect (Figure 4). In other words, the use of a paper-based visual template decreases task precision in culturally diverse groups. This finding is surprising with respect to the extant literature: posters and sticky-notes are often theorized as being beneficial for discussions, regardless of the cultural diversity of groups. It also calls into question current practices in visual facilitation, in which group moderators use visual templates without an adequate consideration of whether they are appropriate given the cultural diversity of groups.

It can be argued that this paradoxical effect is due to the paper-based visual template offering less structuration for group discussion, as compared to the same template loaded onto a collaborative system. Thus, individuals have different opinions as to how the visual templates should be appropriated in practice, which results in decreased precision in addressing the task at hand. Further investigation into the participants' impression of the meeting, collected during the debriefing session, revealed that conflict arose around the enactment of the visual in the paperbased condition. Group members split along the organizational boundary and attempted to take control of the visual template. It was reported that some group members "invaded" the space on the template assigned to their company by placing sticky notes outside the boundaries of their visual space. It seems that the suggestive nature of the paper-based visual template is not constraining and thus does not structure the interaction sufficiently for a multicultural audience.

\section{Discussion}

\section{Theoretical contributions}

Following the perspective of Positive Organizational Scholarship (POS), this study considers cultural diversity as an asset rather than a burden (Stahl and Tung, 2015), and pays attention to the enablers of positive outcomes (Cameron et al., 2003). It contributes to POS by including the visual as a mode of communication that had thus far been neglected, and by articulating the conditions under which such a mode delivers positive outcomes. While several POS studies focus on the 
individual (Shih, 2004) or organizational levels ( $\mathrm{Ng}$ and Tung, 1998; Nielsen and Nielsen, 2013; Stahl and Tung, 2015), we explore the mid-ground of organizational groups - acknowledging that work is increasingly carried out in groups characterized by cultural diversity (Stahl et al., 2010a). And while most POS research has adopted a process perspective to expose the enablers of positive phenomena as they occur in an organization, we used an experimental approach to manipulate the conditions by which culturally diverse groups can benefit from the use of visual templates. The adoption of an experimental approach enables us to establish the internal validity of our findings, and to rule out alternative explanations that may have influenced group performance. POS is pursued further, by venturing towards the adoption of a research approach that has thus far been overlooked in this research tradition (in spite of the recognition of its potential, see van Witteloostuijn, 2015).

This research has the additional merit of developing an interdisciplinary perspective on the study of group cultural diversity. Insights from information systems and visualization are interwoven to elaborate a positive approach that unleashes the potential of culturally diverse groups. In particular, AST (Poole and DeSanctis, 1990) is applied in order to contribute to cross-cultural management and in particular to POS. This interdisciplinary work enables us to account for the main effects produced by the technical system (in this case, the paper-based visual template or the collaborative system) as well as the moderating effect produced by group cultural diversity. This shows that technical and social factors interplay in shaping the extent to which groups produce solutions that are both effective and efficient in addressing the problem at hand.

This work speaks also to scholars interested in the visual and in information systems, by offering insights from an application domain that has not previously been considered in such disciplines, namely that of group cultural diversity. Current literature on the use of visuals in organizations (Meyer et al., 2013) does not consider the effect of group cultural composition, as it assumes that visuals will have the same effect regardless of discussants' background. Conversely, the results of this study clearly indicate that group cultural composition is a relevant contextual factor to consider 
when designing interventions aimed at sustaining group performance. It confirms that culturally diverse members make sense of visual templates differently (Nisbett, 2003), and adds that they need unambiguous support that prevents meta-discussions about how to use a particular visual.

With respect to studies of information systems, our work extends the findings of Staples and Zhao (2006), who compared face-to-face and virtual teams, but did not consider how cultural diversity intertwine with the use of technology in virtual teams.. It also provides initial empirical evidence of Vyas et al.'s (2016) assumption that people perceive different affordances from an information system, depending on their culture. It extends this, by showing how such differences are not negative, but rather enhance the positive effects of collaborative systems on task performance. It also adds that collaborative systems are required in order to provide structuration to the work of culturally diverse groups and prevent group members from engaging in off-topic conversations.

An additional value of this work lies in the fact that experimental groups were highly multicultural, with participants from 49 countries working face-to-face: such highly multicultural co-located groups are seldom investigated, especially in experimental settings.

\section{Contributions to practice}

This work has implications for managerial practice, indicating that a visual template embedded in a collaborative system is an effective way for increasing task precision, especially in culturally diverse, co-located groups. Managers and meeting facilitators are advised to utilize visual templates appropriate for the specific task they are working on, to facilitate their meetings, in order to substantially increase task precision. Visual templates help to overcome multicultural group work challenges, contributing positively to group outcomes. The findings of this study help organizations to leverage the potential of multicultural groups by proposing concrete suggestions to increase their task precision (specifically in the context of strategic alliances' team meetings). Despite the popularity of paper-based templates, posters and sticky notes in organizational group work and in 
facilitators' toolboxes (Sibbet, 2010), this study shows that highly culturally diverse groups might not benefit from these techniques. In culturally diverse groups, a visual template providing higher structuration should be preferred, in order to avoid ambiguity and conflict on how the visual template should be used. Multinational corporations that require their culturally diverse employees to work together should consider the adoption of visual templates embedded in collaborative systems.

Software designers and developers can also derive useful insights from this study: When designing meeting facilitation software, they should consider including visual templates suitable for different tasks (e.g., for mapping group dialogue, documenting participants' contributions and providing guidance in relation to the meeting agenda). In particular, the system should guide users toward a faithful use of the software, so as to avoid different or conflicting interpretations among culturally diverse users.

\section{Limitations and Future Research}

\section{Directions for future research}

Future studies should investigate the potential moderating effects of group (cultural) diversity on different tasks and related visual supports, such as creativity, decision making and productivity ( $\mathrm{Ng}$ and Tung, 1998). A high structuration might be beneficial only for supporting convergent tasks such as precision or decision making, but could be detrimental for divergent tasks such as creativity and innovation. Visual templates with high structuration, in fact, may constrain knowledge sharing and development, by 'locking' participants into the cognitive categories that are made visible by their graphical structure.

As most lab experiments, this study has high internal validity but lower external validity $(\mathrm{Ng}$ and Tung, 1998). Replicating the study in the field could provide a solution to this issue (although its execution may not be free from challenges). This experiment has assessed the effect of visual templates at a given point in time: future researchers may adopt a qualitative approach to explore 
the relationship between visual templates and group cultural diversity over a longer time frame, and possibly for the entire group life-cycle or duration of a project. Since this study has established that the use of visual templates leads to higher task precision, future research may investigate how such templates are enacted in naturally occurring groups - both culturally homogeneous and diverse. This work could lead to additional insights into contextual factors and team dynamics that may impact on the effectiveness of the visual templates by taking a process perspective (Stahl et al. 2010a). Interested researchers may adopt an approach similar to the one of Hajro et al. (2015), who qualitatively investigated knowledge exchange processes of culturally diverse teams.

This study focused on small co-located groups of about four participants. As group size can have a significant effect on performances, future studies should investigate the performances of larger groups. Furthermore, this study concentrated on co-located meetings since these are most common in organizational practice, but exploration of cultural diversity and visual facilitation in the context of dislocated meetings (e.g., online) is also encouraged. Future research could also make cultural diversity an experimental condition, and assess whether subjects are multicultural individuals (Fitzsimmons et al., 2011) - as this could influence the measurement of group cultural diversity.

Finally, cultural diversity has been operationally defined by computing an index of diversity based on group members' place of origin with respect to the world macro regions. Given the paucity of studies on highly culturally diverse groups and the lack of consensus on how to measure cultural diversity (Shenkar, 2001), our study has conceptualized and operationalized the degree of group cultural diversity based on world macro regions. To investigate more deeply the underlying mechanisms of the moderating role of culture, future studies could apply more refined measures of group cultural diversity by operationalizing it with comparative culture frameworks such as GLOBE (House et al., 2004; House et al,. 2013). An alternative option is to consider espoused cultural values (Srite and Karahanna, 2006; Hoehle et al., 2015); although these may be difficult to assess in an experimental study. 


\section{Concluding remarks}

In line with POS, the purpose of this study was to investigate "enablers" of positive outcomes in organizations (e.g., structures and systems), as defined by Stahl et al. (2010a, pg. 441). In particular, we have focused on visual templates as enablers of positive group outcomes. The hypotheses find confirmation in the data: 1) visual templates that provide higher structuration improve task precision in meetings; and 2) group cultural diversity positively moderates the relationship between structuration and task precision. In the presence of higher structuration, culturally diverse groups are able to productively leverage their differences in cognitive styles and values, so as to deliver solutions that are both effective and efficient in addressing the task at hand (task precision). On the contrary, visual templates that provide lower structuration sustain task precision only for culturally homogeneous groups. The more culturally diverse the group, the greater the need of providing structuration: a support with lower structuration decreases precision, possibly because of the ambiguity in its interpretation of usage (see also Vyas et al., 2016). These intriguing results call for further investigation to understand the optimal level of structuration required for different levels of cultural diversity and for different tasks. With this research, it is hoped that the management community is stimulated toward the study of visual templates and their effects on culturally diverse group dynamics (which promises to be highly relevant for both theory and practice), and to have contributed to the emergent stream of POS. 


\section{References}

Adler, N. J. (2003), International Dimensions of Organizational Behavior, South-Western College Publishing, Cincinnati, $\mathrm{OH}$.

Alexander, E., Bresciani, S. and Eppler, M. J. (2015), "Understanding the impact of visual representation restrictiveness on experience sharing: An experimental assessment", Journal of Visual Languages and Computing, Vol. 31, PART A, pp. 30-46.

Al-Kassab, J., Ouertani, Z. M., Schiuma, G. And Neely, A. (2014), “Information visualization to support management decisions", International Journal of Information Technology and Decision Making, Vol. 13 No. 02, pp. 407-428.

Baron, R. M. and Kenny, D. A. (1986), "The moderator-mediator variable distinction in social psychological research: Conceptual, strategic, and statistical considerations", Journal of personality and social psychology, Vol. 51 No. 6, pp. 1173-1182.

Berry, J.W. (2015), “Global psychology: implications for cross-cultural research and management”, Cross Cultural Management, Vol. 22 No. 3, pp. 342 - 355.

Blau, P. M. (1977), Inequality and heterogeneity. Free Press. New York, NY.

Bresciani, S. (2013), “Organizational communication with visual mapping: Comparing East and West”, in Ingenhoff, D. (Ed.), Internationale PR-Forschung, UVK Verlag, Konstanz, pp. $37-$ 52.

Bresciani, S. and Eppler, M.J. (2009), "The benefits of synchronous collaborative information visualization: Evidence from an experimental evaluation” IEEE Transactions on Visualization and Computer Graphics, Vol. 15 No. 6, pp. 1073-1080.

Buzan, T. (2004), Mind Maps at Work: How to Be the Best at Your Job and Still Have Time to Play, HarperCollins, London, U.K.

Cabitza, F. and Simone, C. (2013), “Computational coordination mechanisms: A tale of a struggle for flexibility", Computer Supported Cooperative Work (CSCW), Vol. 22 No. 4, pp. 475-529. 
Cameron, K., Dutton, J. and Queen, R.E. (2003), Positive Organizational Scholarship: Foundations of a New Discipline, Berrett-Koehler Publishers, San Francisco, CA.

Campbell, D.T. (1959), "Methodological suggestions from a comparative psychology of knowledge processes", Inquiry, Vol. 2 No. 1-4, pp. 152-182.

Campion, M.A., Medsker, G.J. and Higgs, A.C. (1993), "Relations between work group characteristics and effectiveness: Implications for designing effective work groups", Personnel Psychology, Vol. 46 No. 4, pp. 823-850.

Collard, I. F., and Foley, R. A. (2002), "Latitudinal patterns and environmental determinants of recent human cultural diversity: do humans follow biogeographical rules?" Evolutionary Ecology Research, Vol. 4 No. 3, pp. 371-383.

Comi, A., Eppler, M. J. and Pietroforte, R. (2009), Building Innovation Alliances on Complementary Competences: The Beacon and Dioguardi Case Study. Case study with Teaching Note, ECCH case collection (Ref. no. 309-153-1, 309-154-1, 309-153-8).

Daily B., Whatley, A., Ash, S.R. and Steiner R.L. (1996), “The effects of a group decision support system on culturally diverse and culturally homogeneous group decision making", Information and Management, Vol. 30 No. 6, pp. 281-289.

Demir, R. (2015), "Strategic activity as bundled affordances”, British Journal of Management, Vol. 26, No. 1, pp. S125-S141.

DeSanctis, G. and Poole, M. S. (1994), “Capturing the complexity in advanced technology use: Adaptive structuration theory", Organization Science, Vol. 5 No. 2, pp. 121-147.

Eppler, M. J. and Burkhard, R. A. (2007), "Visual representations in knowledge management: Framework and cases", Journal of Knowledge Management, Vol. 11 No. 4, pp. 112-122. Eppler, M. J. and Platts, K. W. (2009), "Visual strategizing: The systematic use of visualization in the strategic-planning process", Long Range Planning, Vol. 42 No. 1, pp. 42-74. 
Etudo, U., Weistroffer, H. R. and Al-Abdullah, M. (2015), Adaptive structuration theory and media richness theory in GDSS research: A critical review. In $48^{\text {th }}$ Hawaii International Conference on System Sciences (HICSS) 2015 (pp. 344-353). IEEE, HI, USA.

Field, A. (2009), Discovering Statistics Using SPSS, SAGE Publications, London, UK.

Fitzsimmons, S. R., Miska, C. And Stahl, G. K. (2011), “Multicultural employees: Global business' untapped resource", Organizational Dynamics, Vol. 40 No. 3, pp. 199-206.

Geraldi, J. and Arlt, M. (2015), Visuals Matter! Designing and Using Effective Visual Representations to Support Project and Portfolio Decisions. Project Management Institute, Newtown Square, Pennsylvania.

Gibbs, J. P. and Martin, W. T. (1962), "Urbanization, technology, and the division of labor: International patterns”, American Sociological Review, Vol. 27 No. 5, pp. 667-677.

Gibson, J. (1979), The Ecological Approach to Visual Perception, Houghton Mifflin, New York, NY.

Gray, P. and Olfman, L. (1989), "The user interface in group decision support systems", Decision Support Systems, Vol. 5 No.2, pp. 119-137.

Hajro, A., Gibson, C. and Pudelko, M. (2015), "Knowledge exchange processes in multicultural teams: Linking organizational diversity climates to teams' effectiveness", Academy of Management Journal, Published online before print December 14, 2015.

Hoehle, H., Zhang, X. and Venkatesh, V. (2015), “An espoused cultural perspective to understand continued intention to use mobile applications: a four-country study of mobile social media application usability”, European Journal of Information Systems, Vol. 24 No. 3, pp. 337-359.

Hofstede, G. (2001), Culture's Consequences: Comparing Values, Behaviors, Institutions, and Organizations across Nations, SAGE Publications, Thousand Oaks, CA.

Hofstede, G., Hofstede, G. J., Minkov M. and Vinken H. (2008), Values Survey Module 2008 Manual. Geert Hofstede BV. 
House, R.J., Hanges, P.J., Javidan, M., Dorfman, P.W. and Gupta, V. eds., (2004), Culture, leadership, and organizations: The GLOBE study of 62 societies. Sage Publications, Thousand Oaks, CA.

House, R. J., Dorfman, P. W., Javidan, M., Hanges, P. J. And de Luque, M. F. S. (2013), Strategic Leadership across Cultures: GLOBE Study of CEO Leadership Behavior and Effectiveness in 24 Countries, SAGE Publications, Thousand Oaks, CA.

Hundhausen, C. (2005), "Using end user visualization environments to mediate conversations: A ‘Communicative Dimensions' framework”, Journal of Visual Languages \& Computing, Vol. 16 No. 3, pp. 153-185.

Isenberg, P., Elmqvist, N., Scholtz, J., Cernea, D., Ma, K. L. and Hagen, H. (2011), “Collaborative visualization: definition, challenges, and research agenda", Information Visualization, Vol. 10 No. 4, pp. 310-326.

Jackson, S.E., Joshi, A. and Erhardt, N.L., (2003), "Recent research on team and organizational diversity: SWOT analysis and implications", Journal of Management, Vol. 29 No. 6, pp. 801-830.

Jarzabkowski, P. and Kaplan, S. (2015), "Strategy tools-in-use: A framework for understanding “technologies of rationality” in practice", Strategic Management Journal, Vol. 36 No. 4, pp. $537-558$.

Jones, M.R. and Karsten, H., (2008), “Giddens's structuration theory and information systems research", MIS Quarterly, Vol. 32 No. 1, pp.127-157.

Judd, C. M., Yzerbyt, V. and Muller, D. (2014), "Mediation and moderation", in Reis, H. and Judd, C.M. (Eds.), Handbook of Research Methods in Personality and Social Psychology, Cambridge University Press, New York, NY, pp. 653-676,

Leung, K. and Morris, M., (2015), "Values, Schemas, and Norms in the Culture-Behavior Nexus: A Situated Dynamics Framework”, Journal of International Business Studies, Vol. 46 No. 9, pp. 1028-1050. 
Levine, R. N. (2008). A Geography of Time: On Tempo, Culture, and the Pace of Life. Basic Books.

Mach, M. and Baruch, Y. (2015), "Team performance in cross cultural project teams: The moderated mediation role of consensus, heterogeneity, faultlines and trust", Cross Cultural Management: An International Journal, Vol. 22 No. 3, pp. 464-486.

Markus, H. R. and Kitayama, S. (1991), “Culture and the self: Implications for cognition, emotion, and motivation", Psychological Review, Vol 98 No. 2, pp. 224-253.

Mathieu, J., Maynard, M. T., Rapp, T. and Gilson, L. (2008), “Team effectiveness 1997-2007: A review of recent advancements and a glimpse into the future", Journal of Management, Vol. 34 No. 3, pp. 410-476.

McLeod, P. L., Lobel, S. A. and Cox, T. H. (1996), "Ethnic diversity and creativity in small groups", Small Group Research, Vol. 27 No. 2, pp. 248-264.

Mell J., van Knippenberg D. and van Ginkel W. (2013), "The catalyst effect: the impact of transactive memory system structure on group performance", Academy of Management Journal, Vol. 57 No. 4, pp. 1154-1173.

Meyer, E. (2014). The Culture Map. Breaking through the Invisible Boundaries of Global Business, PublicAffairs, New York, NY.

Meyer, R. E., Höllerer, M. A., Jancsary, D. And Van Leeuwen, T. (2013), "The visual dimension in organizing, organization, and organization research: Core ideas, current developments, and promising avenues", The Academy of Management Annals, Vol. 7 No. 1, pp. 489-555.

Milliken, F. and Martins, L. (1996), "Searching for common threads: Understanding the multiple effects of diversity in organizational groups", Academy of Management Review, Vol. 21 No. 2, pp. 402-433.

Muller, A. And Välikangas, L. (2002), "Extending the boundary of corporate innovation”, Strategy and Leadership, Vol. 30 No. 3, pp. 4-9. 
Naik, N. And Kim, D. J. (2010), “An Extended Adaptive Structuration Theory for the determinants and Consequences of Virtual Team Success", In: ICIS 2010 Proceedings, International Conference on Information Systems Paper 232.

Ng, E. S. and Tung, R. L. (1998), "Ethno-cultural diversity and organizational effectiveness: A field study”, International Journal of Human Resource Management, Vol. 9 No. 6, pp. 980-995.

Niederman, F., Briggs, R. O., de Vreede, G. J. and Kolfschoten, G. L. (2008), "Extending the Contextual and Organizational Elements of Adaptive Structuration Theory in GSS Research”, Journal of the Association for Information Systems, Vol. 9 No. 10/11, pp. 633- 652.

Nielsen, B.B. and Nielsen, S., (2013), “Top management team nationality diversity and firm performance: A multilevel study”, Strategic Management Journal, Vol. 34 No. 3, pp. 373382.

Nisbett, R. E. (2003), The Geography of Thought, Free Press, New York, NY.

Norenzayan, A., Smith, E. E., Kim, B. J. and Nisbett, R. E. (2002), "Cultural preferences for formal versus intuitive reasoning”. Cognitive Science, Vol. 26 No. 5, pp. 653-684.

Pattanaik, D. (2016), Olympus. An Indian Retelling of the Greek Myths. Penguin Random House India.

Paul, S., Seetharaman, P., Samarah, I. And Mykytyn, P. P. (2004), "Impact of heterogeneity and collaborative conflict management style on the performance of synchronous global virtual teamgroups", Information and Management, Vol. 41 No. 3, pp. 303-321.

Perez Garcia, M. and Bresciani, S. (2015), “The Role of Visual Templates on Improving Teamwork Performance”. IEEE Proceedings of the IV 2015 - $19^{\text {th }}$ International Conference Information Visualisation, 21-24 July 2015, Barcelona, Spain.

Pietroforte, R. (1996), Building International Construction Alliances. Successful Partnering for Construction Firms, E \& FN Spon, Boundary Row, London, UK. 
Poole, M. S. and DeSanctis, G. (1990), “Understanding the use of group decision support systems: The theory of adaptive structuration", in Fulk J. and Steinfeld C. (Eds), Organizations and Communication Technology, SAGE Publications, Newbury Park, CA, pp. 173-193.

Roam, D. (2009), The Back of the Napkin: Solving Problems and Selling Ideas with Pictures, Penguin Group, New York, NY.

Schnotz, W. and Kurschner, C. (2008), "External and internal representations in the acquisition and use of knowledge: visualization effects on mental model construction”, Instructional Science, Vol. 36 No. 3, pp. 175-190.

Schulz-Hardt, S., Brodbeck, F., Mojzisch, A., Kerschreiter, R. and Frey, D. (2006), "Group decision making in hidden profile situations: Dissent as a facilitator for decision quality", Journal of Personality and Social Psychology, Vol. 91 No. 6, pp. 1080-1093.

Shachaf, P. (2008), "Cultural diversity and information and communication technology impacts on global virtual teams: An exploratory study", Information and Management, Vol. 45 No. 2, pp. $131-142$

Shenkar, O. (2001), “Cultural distance revisited: Towards a more rigorous conceptualization and measurement of cultural differences" Journal of International Business Studies, Vol. 32 No. 3, pp. 519-535.

Shenkar, O., Luo, Y. And Yeheskel, O. (2008), "From "distance" to "friction": Substituting metaphors and redirecting intercultural research", Academy of Management Review, Vol. 33 No. 4, pp. 905-923.

Shigenobu, T., Fujii, K. and Yoshino, T. (2007), "The role of annotation in intercultural communication", in Aykin, N., Usability and Internationalization. HCI and Culture, Springer, Berlin, Germany, pp. 186-195.

Sibbet, D. (2010), Visual Meetings: How Graphics, Sticky Notes and Idea Mapping Can Transform Group Productivity. John Wiley \& Sons, Hoboken, New Jersey, NJ. 
Shadish, W.R., Cook, T. D. and Campbell, D. T. (2002), Experimental and quasi-experimental designs for generalized causal inference. Wadsworth Cengage Learning, Boston and New York.

Shih, M. 2004. Positive stigma: Examining resilience and empowerment in overcoming stigma. Annals of the American Academy of Political and Social Science, Vol. 591 No. 1, pp. 175185.

Silver, M. S. (2008), “On the design features of decision support systems: The role of system restrictiveness and decisional guidance", in Burstein, F. and Holsapple, C. W. (Eds.), Handbook on Decision Support Systems 2: Variations, Springer, Berlin Heidelberg, pp. 261291.

Snell, B. (1953) “The Discovery of the Mind: The Greek Origins of European Thought". Harvard University Press, Cambridge, MA.

Srite, M. And Karahanna, E. (2006), “The role of espoused national cultural values in technology acceptance", MIS Quarterly, Vol. 3 No. 3, pp. 679-704.

Stahl, G. K. and Tung, R. L. (2015), “Towards a more balanced treatment of culture in international business studies: The need for positive cross-cultural scholarship", Journal of International Business Studies, Vol. 46 No. 4, pp. 391-414.

Stahl, G. K., Mäkelä, K., Zander, L. And Maznevski, M. L. (2010a), "A look at the bright side of team diversity", Scandinavian Journal of Management, Vol. 26 No. 4, pp. 439-447.

Stahl, G. K., Maznevski, M. L., Voigt, A. and Jonsen, K. (2010b), "Unraveling the effects of cultural diversity in teams: A meta-analysis of research on multicultural work groups", Journal of International Business Studies, Vol. 41 No. 4, pp. 690-709.

Staples, D. S. and Zhao, L. (2006), "The effects of cultural diversity in virtual teams versus face-toface teams", Group Decision and Negotiation, Vol. 15 No. 4, pp. 389-406.

Stasser, G. and Titus, W. (2003), "Hidden profiles: A brief history”, Psychological Inquiry, Vol. 14 No. 3-4, pp. 304-313. 
Suthers, D.D. (2001), “Toward a systematic study of representational guidance for collaborative learning discourse", Journal of Universal Computer Science, Vol. 7 No. 3, pp. 254-277.

Taras, V., Rowney, J. and Steel, P. (2009), "Half a century of measuring culture: Review of approaches, challenges, and limitations based on the analysis of 121 instruments for quantifying culture”, Journal of International Management, Vol. 15 No. 4, pp. 357-373.

Tomlinson, J. (1999). "Globalization and culture". The University of Chicago Press Books. Chicago, IL.

Tung, R. L. And Verbeke, A. (2010), "Beyond Hofstede and GLOBE: Improving the quality of cross-cultural research", Journal of International Business Studies, Vol. 41 No. 8, pp. 12591274.

Van Knippenberg, D., De Dreu, C. K. and Homan, A. C. (2004), "Work group diversity and group performance: an integrative model and research agenda", Journal of Applied Psychology, Vol. 89 No, 6, pp. 1008-1022.

Van Witteloostuijn, A. (2015), “Toward Experimental International Business: Unraveling Fundamental Causal Linkages”, Cross Cultural Management, Vol. 22 No. 4, pp. 530-544.

Vyas, D., Chisalita, C. M. and Dix, A. (2016), “Organizational Affordances: A Structuration Theory Approach to Affordances", Interacting with Computers. First published online: May 4, 2016.

Watson, R. T., Ho, T. H. And Raman, K. S. (1994), "Culture: A fourth dimension of group support systems", Communications of the ACM, Vol. 37 No. 10, pp. 44-55.

Wheeler, B. C. and Valacich, J. S. (1996), "Facilitation, GSS, and training as sources of process restrictiveness and guidance for structured group decision making: An empirical assessment”, Information Systems Research, Vol. 7 No. 4, pp. 429-450.

Williams, K. Y. and O’Reilly, C. A. (1998), “Demography and diversity in organizations: A review of 40 years of research", Research in Organizational Behavior, Vol. 20, pp. 77-140.

Yakura, E.K., (2002), “Charting Time: Timelines as Temporal Boundary Objects”, Academy of Management Journal, Vol. 45 No. 5, pp. 956-970. 
Yates, J. and Orlikowski, W. (2007), “The PowerPoint presentation and its corollaries: How genres shape communicative action in organizations", in Zachry, M. and Thralls, C. (Eds.), Communicative Practices in Workplaces and the Professions: Cultural Perspectives on the Regulation of Discourse and Organizations, Baywood Publishing, Amityville, NY, pp. 6791.

Zhang, D., Lowry, P. B., Zhou, L. and Fu, X. (2007), “The impact of individualism-collectivism, social presence, and group diversity on group decision making under majority influence", Journal of Management Information Systems, Vol. 23 No. 4, pp. 53-80.

Zoogah, D. B., Vora, D., Richard, O. and Peng, M. W. (2011), "Strategic alliance team diversity, coordination, and effectiveness", The International Journal of Human Resource Management, Vol. 22 No. 3, pp. 510-529. 
Table 1. Correlations among Observed and Control Variables

\begin{tabular}{|c|c|c|c|c|c|c|c|c|c|c|c|}
\hline & 1 & 2 & 3 & 4 & 5 & 6 & 7 & 8 & 9 & 10 & 11 \\
\hline $\begin{array}{l}\text { 1. Structuration of } \\
\text { visual templates }\end{array}$ & - & & & & & & & & & & \\
\hline $\begin{array}{l}\text { 2. Group cultural } \\
\text { diversity }\end{array}$ & -.04 & - & & & & & & & & & \\
\hline 3. Task precision & $.63^{* *}$ & -.08 & - & & & & & & & & \\
\hline 4. Gender & -.11 & -.06 & -.04 & - & & & & & & & \\
\hline 5. Age & $-.21^{* *}$ & .10 & -.30 & .15 & - & & & & & & \\
\hline $\begin{array}{l}\text { 6. Experience of } \\
\text { strategic alliances }\end{array}$ & .02 & -.03 & .05 & -.04 & $-.26^{* *}$ & - & & & & & \\
\hline $\begin{array}{l}\text { 7. Level of } \\
\text { English language }\end{array}$ & -.07 & $-.24^{* *}$ & -.05 & -.01 & -.12 & $.19^{*}$ & - & & & & \\
\hline $\begin{array}{l}\text { 8. Preference for } \\
\text { group work }\end{array}$ & -.03 & -.08 & -.05 & .04 & $.15^{*}$ & .04 & .09 & - & & & \\
\hline $\begin{array}{l}\text { 9. Preference for } \\
\text { visualization }\end{array}$ & -.08 & $-.14^{*}$ & -.04 & .01 & .04 & .12 & .09 & $.25^{* *}$ & - & & \\
\hline $\begin{array}{l}\text { 10. Facilitator's } \\
\text { skillfulness }\end{array}$ & .06 & -.06 & -.01 & $-.13^{*}$ & .02 & .05 & 0.5 & .07 & .03 & - & \\
\hline $\begin{array}{l}\text { 11. Number of } \\
\text { group participants }\end{array}$ & -.09 & .00 & $-.15 *$ & -.05 & $.19 * *$ & .02 & .04 & .03 & .09 & .11 & - \\
\hline
\end{tabular}

* Correlation is significant at the 0.05 level (2-tailed).

** Correlation is significant at the 0.01 level (2-tailed). 
Table 2. Descriptive statistics stratified per condition

\begin{tabular}{|c|c|c|c|c|}
\hline Condition & $\begin{array}{l}\text { Group Cultural } \\
\text { Diversity }^{\mathrm{a}}\end{array}$ & $\begin{array}{l}\text { Mean Value of } \\
\text { Precision }^{\mathrm{b}}\end{array}$ & $\mathbf{N}$ & S. D. \\
\hline \multirow{7}{*}{$\begin{array}{l}\text { No structuration } \\
\text { (No visual templates) }\end{array}$} & 0.00 (homogeneous) & .6183 & 6 & .177 \\
\hline & 0.33 & .4900 & 1 & \\
\hline & 0.50 & .7983 & 6 & .131 \\
\hline & 0.67 & .3900 & 2 & .197 \\
\hline & 0.80 & .8200 & 1 & \\
\hline & 0.83 & .7000 & 3 & .170 \\
\hline & Full sample & .6679 & 19 & .192 \\
\hline \multirow{6}{*}{$\begin{array}{l}\text { Lower structuration } \\
\text { (Paper-based visual templates) }\end{array}$} & 0.00 (homogeneous) & .8400 & 7 & .121 \\
\hline & 0.40 & .6400 & 1 & \\
\hline & 0.50 & .7367 & 6 & .097 \\
\hline & 0.67 & .6833 & 3 & .205 \\
\hline & 0.83 & .7400 & 3 & .036 \\
\hline & Full sample & .7605 & 20 & .126 \\
\hline \multirow{6}{*}{$\begin{array}{l}\text { Higher structuration (Visual } \\
\text { templates in a collaborative system) }\end{array}$} & 0.00 (homogeneous) & .9388 & 8 & .071 \\
\hline & 0.50 & .9275 & 4 & .055 \\
\hline & 0.67 & .9200 & 1 & \\
\hline & 0.83 & .9233 & 3 & .080 \\
\hline & 1.00 & .9600 & 1 & \\
\hline & Full sample & .9335 & 17 & .061 \\
\hline
\end{tabular}

${ }^{a}$ A value of 0.00 indicates that all group members are from the same world region (that is, a culturally homogeneous group); 1.00 indicates that all group members are from a different world region.

${ }^{\mathbf{b}}$ Precision is calculated as the ratio of correct items over the total number of items identified. A value of 1 represents the highest possible precision (when all items identified by a group are correct - according to the case solution). 
Table 3. Significance testing of the moderation of support' structuration and group cultural diversity on precision

\begin{tabular}{|l|l|l|l|}
\hline Variables: & F & df & Sig. \\
\hline Structuration of visual templates & 8.488 & 2 &. $.016 *$ \\
\hline Group cultural diversity & 0.741 & 8 & .660 \\
\hline Structuration of visual templates * group cultural diversity & 7.432 & 6 & $\mathbf{. 0 0 0} * *$ \\
\hline
\end{tabular}

*Significance at $<.05$ level, two tailed $\quad * *$ Significance at $<.001$ level, two tailed

Table 4. Contrasts of the main effect of structuration of the support on precision

\begin{tabular}{|l|l|l|l|}
\hline Contrasts & Mean & St. Error & Sig. \\
& difference & & \\
\hline Control (no visual templates) vs. paper-based visual templates & -.0911 & .01930 & $\mathbf{. 0 0 0}^{* * *}$ \\
\hline $\begin{array}{l}\text { Paper-based visual templates vs. visual templates in a } \\
\text { collaborative system }\end{array}$ & & &. .1801 \\
\hline
\end{tabular}

*Significance at $<.05$ level, two tailed $\quad * *$ Significance at $<.001$ level, two tailed 
Table 5. ANCOVA with all control variables added to the model simultaneously

\begin{tabular}{|c|c|c|c|}
\hline & $\mathbf{F}$ & df & Sig. \\
\hline Age & 6.399 & 1 & $.012 *$ \\
\hline Gender & 0.184 & 1 & .668 \\
\hline Preference for group work & 0.066 & 1 & .798 \\
\hline Preference for visualization & 0.062 & 1 & .804 \\
\hline Facilitator's skillfulness & 0.248 & 1 & .619 \\
\hline Level of English language & 0.040 & 1 & .843 \\
\hline Experience of strategic alliances & 0.001 & 1 & .981 \\
\hline Number of group participants & 0.008 & 1 & .929 \\
\hline Structuration of visual templates (main effect) & 32.887 & 2 & $.000 * *$ \\
\hline Group cultural diversity (moderator) & 2.834 & 8 & $.005 *$ \\
\hline $\begin{array}{l}\text { Structuration of visual templates } * \text { group cultural } \\
\text { diversity (moderation) }\end{array}$ & 5.210 & 6 & $.000 * *$ \\
\hline
\end{tabular}

*Significance at <.05 level, two tailed $\quad * *$ Significance at $<.001$ level, two tailed 


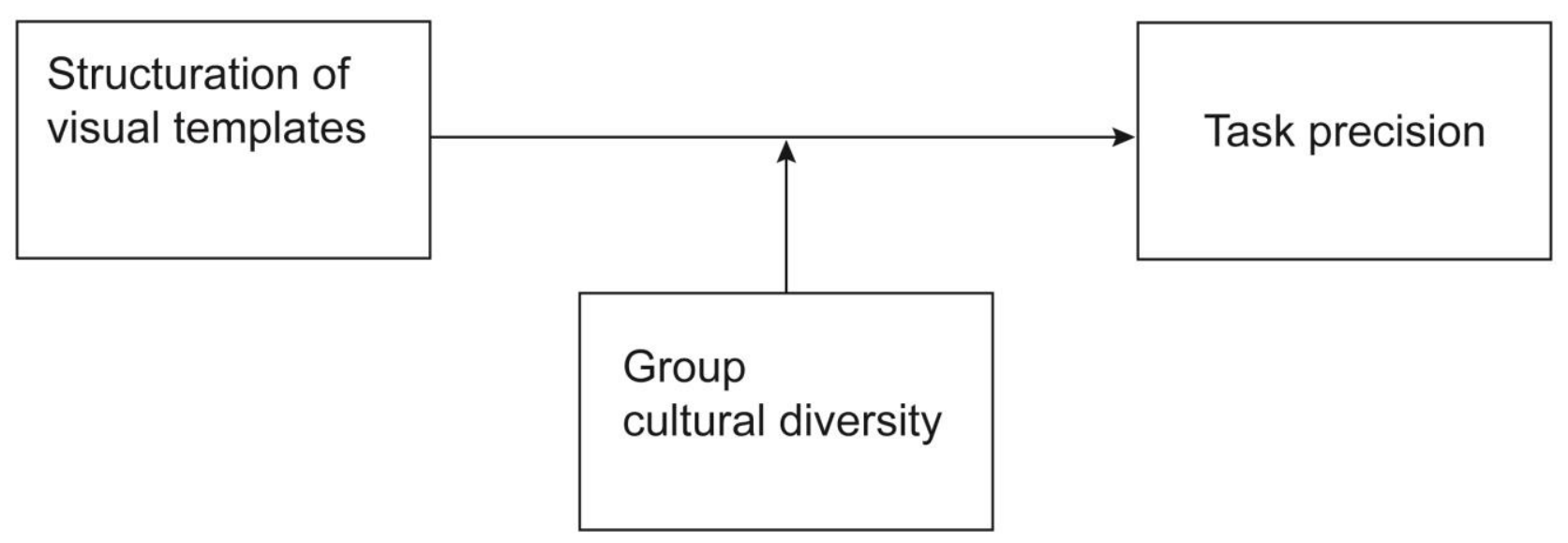

Figure 1. Research model

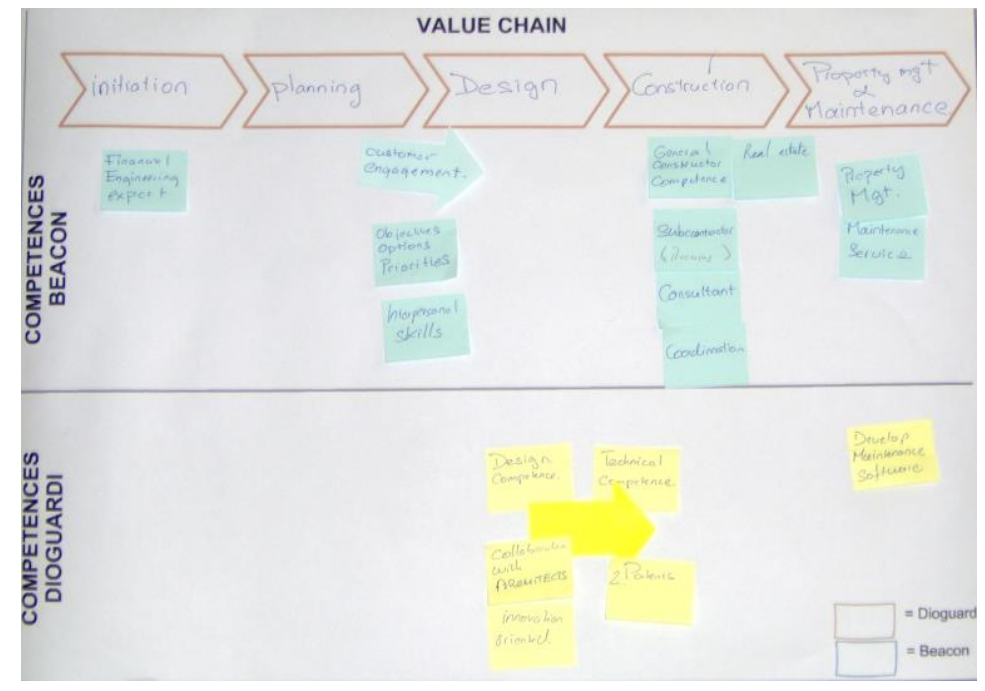

Figure 2. The results of a group working with the competence complementarity chain template in the condition of lower structuration (paper-based visual templates) 


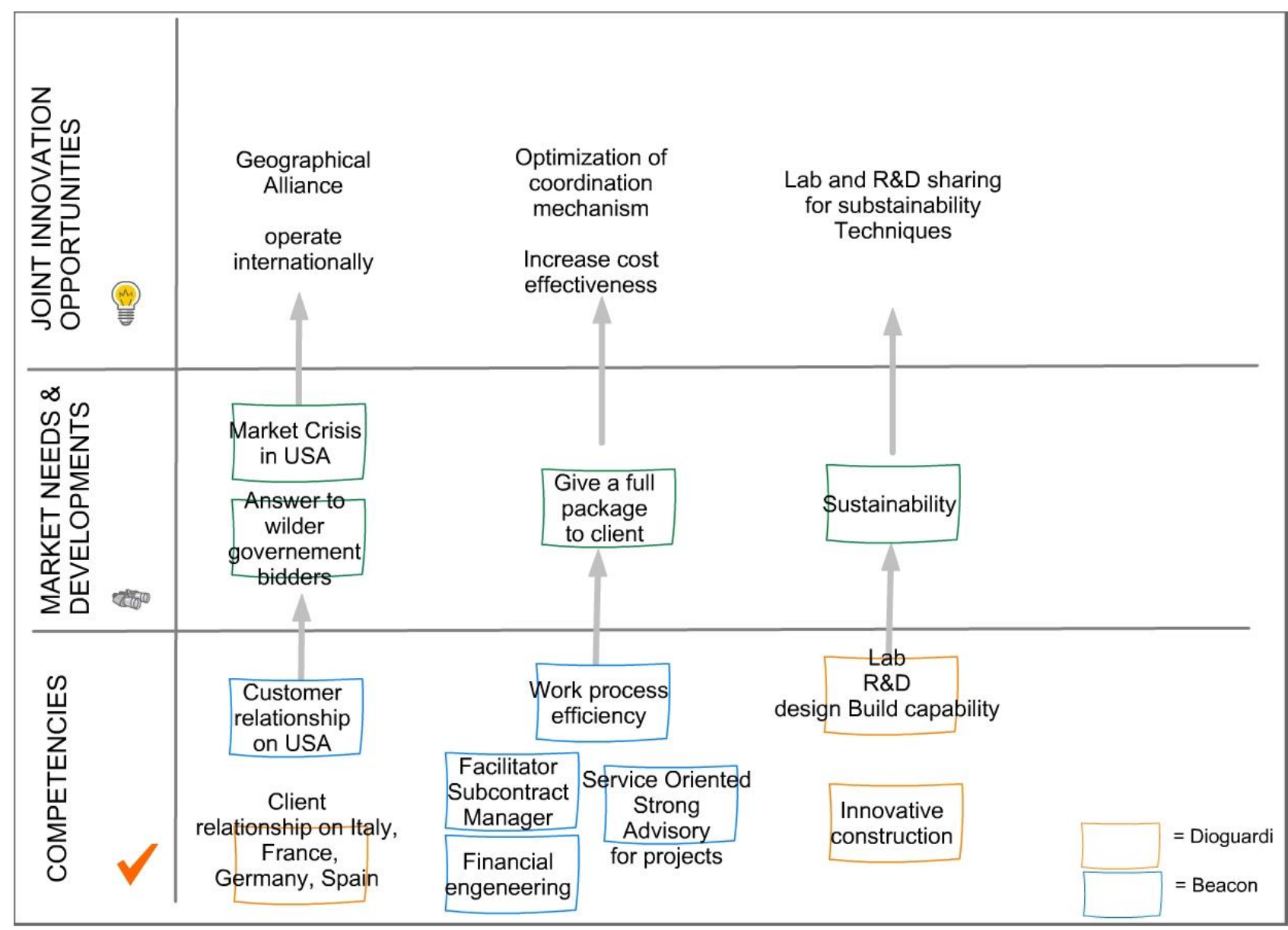

Figure 3. The results of a group working with the innovation opportunity map in the condition of higher structuration (visual templates on collaborative system) 


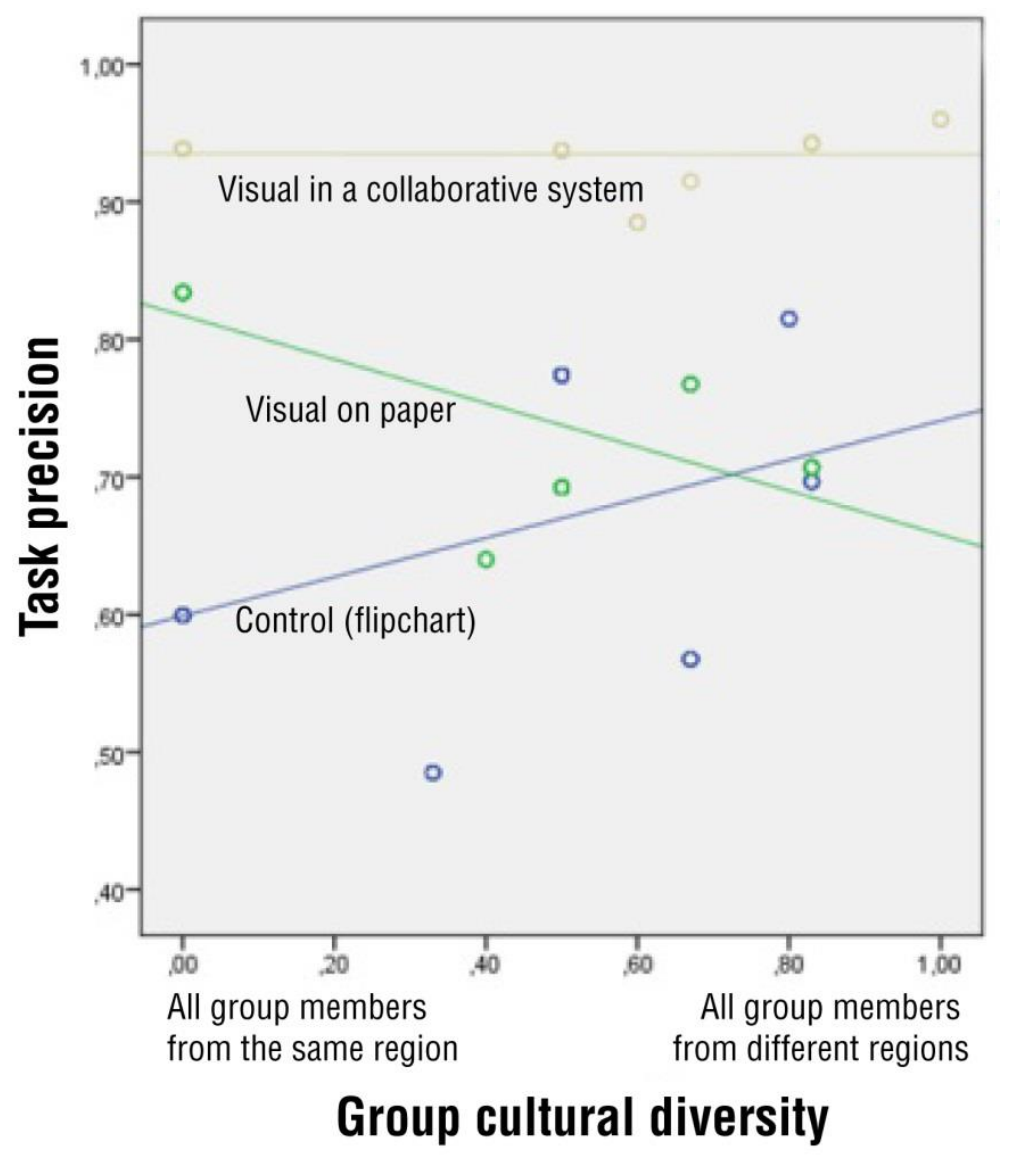

Figure 4. The moderation effect of cultural diversity 